\title{
The Definite Form of the Coxa Plana
}

\author{
by \\ Henning Waldenström, M. D. \\ (Tabulae XXVI-XXX)
}

First a few words must be said about the name coxa plana; it is relatively new. It was suggested by the author in 1920 . The name refers to the most characteristic attributes of the disease, viz., that the caput, collum and acetabulum, that is to say, the entire hip-joint becomes flattened. The name is taken to be analogous with coxa vara and coxa valga, the two other big groups of deformities in the hip; and just as in the case of coxa vara, where one speaks of a coxa vara essentialis, an osteomyelitica, a tuberculotica, and so on, one can speak of a coxa plana essentialis, an osteomyelitica, a tuberculotica, etc. Without a doubt the most usual of these, and the only one which will here be described, is the coxa plana essentialis, here, for the sake of brevity, called coxa plana. In the literature the disease is called Legg's disease, maladie de Calvé, Perthes Krankheit, and so on, and osteochondritis deformans coxae juvenilis. The first names are unsuitable because they are the names of persons. The last name is incorrect for it is not a question of an inflammation.

At the beginning most of those who described the disease believed that the cause was due to some bacterial influence; Legg and Perthes believed in staphylococci and the writer believed that some benign form of tuberculosis was present. Under such circumstances the name of osteochondritis might be accounted for; but now, when it is known that the cause of coxa plana essentialis is not due to any inflammatory influence, it becomes misleading.

Can a coxa plana essentialis be differentiated from a coxa plana tuberculosa, staphylomycotica, etc. with certainty on a roentgen picture during the stage of development? No, it cannot. These coxa plana of inflammatory origin are very rare, but they are nevertheless to be found. I reported in my publication of 1909 a case of certain tuberculous coxa plana which, at a certain stage, both with respect to symptoms and the 
roentgen picture, could not be differentiated from the coxa plana essentialis. The development afterwards became a tuberculosis in the whole joint. I have likewise had a case of coxa plana after a rheumatic inflammation in the hip-joint under observation which, also, at a certain stage of its development, resembled exactly a coxa plana essentialis. This will be published in the Archive franco-belge 1922.

In order to be able to say with certainty, in a positive case of coxa plana, that it is an essentialis, a certain observation period is sometimes needed, so that the typical development can be seen, together with an anamnesis that does not suggest an inflammatory process. Nevertheless, these cases of coxa plana non essentialis are surely so rare that, in practice, one can generally make a diagnosis of a coxa plana essentialis in the stadium of its development, without needing any observation period.

LEGG and the writer were the first to describe this disease; it was in 1909. The following year it was described by Sourdat, Calvé and Perthes, in the order just given. Since this, numberless publications have dealt with this disease. I will not give any review of these works, but will simply mention a few words about that which is found in the literature respecting the final stage. No one as yet has attempted to explain the final stage; only single cases are reported where the authors believe that, from the appearance of the deformity in a certain case, they can infer a coxa plana in the final stage. Thus Sinding-Larsen, Sundt, LegG, Taylor and Frieder, Perthes, Schwartz, Nové-Josserand, PANNER and the writer have reported such cases. Some of these cases have doubtless never been coxa plana. LEGG has divided the final stage into two groups, according to the form, viz., »cap-deformity» and "mushroom deformity». His cases, however, have not been under observation from the beginning of the disease and they, therefore, do not decide the actual, definite form. SUNDT alone, by means of after-examination of some of SiNDING-LARSEN's cases, has been enabled to describe roentgenograms from a period when the development was concluded and which, therefore, must be regarded as definite.

This paper is thus intended to endeavour to clear up by demonstrated cases of coxa plana the hitherto rather obscure question as to the appearance of the definite form of coxa plana. For this it is necessary, firstly, to have observed the beginning and the development of the disease, so that every case included is, with certainty, a coxa plana essentialis; secondly, these cases must, moreover, be kept under observation for so long a period that the normal growth is concluded; and thirdly, a sufficiently large number of cases must have been under observation, so that the typical kind of all the varying forms that the coxa plana can assume may be ascertained. That this careful method of pro- 
cedure is necessary, when the determination of the definite form of coxa plana is aimed at, is shown, among other things, by a case of tuberculous coxa plana represented by the roentgenograms (Fig. 10). On the first picture a typical tuberculous coxitis is seen (Fig. 10 a) with the joint atrophic, the articulatory cartilage thinned, and the clinical symptoms of a tuberculous coxitis. Ten years afterwards it is shown on the roentgen picture (Fig. 10 b) as a typical coxa plana, a coxa plana tuberculosa, impossible now, in the definite form, to see any tuberculous character. Since 1907, when I observed my first case of coxa plana, I have treated and carefully roentgen-examined 37 cases of coxa plana essentialis. Five of these are double-sided, therefore the number of hip-joints is 42. Of these, 32 were boys and 5 girls.

In 22 of these hip-joints I have followed the development from the beginning until the growth was concluded. Out of these 22 there are 10 who have exceeded the age of 20 years. The form of the hip for many years has been the same. My opinion is that the definite form is reached before the conclusion of growth. It is these 22 cases which serve as a basis for the conception respecting the final form that I here advance.

Coxa plana offers interest especially from a roentgenological point of view. It not often yields symptoms that demand special treatment. Sometimes there are pains and stiffness and then it is best to prescribe rest in bed, or plaster of Paris for a longer or shorter period. But the treatment is of no real importance for the development of the disease. My cases have been treated in different ways, sometimes by rest in bed, or extension, or immobilisation bandages, etc. without the disease appearing to have been influenced in its development. It develops quite in the same typical and presumably predestined manner whatever one does. Perhaps great exertions should be forbidden (gymnastics, jumping, football, and so on.). One of my most severe cases, in respect to the extent of the deformity, was treated the whole time with rest in bed and permanent extension, and afterwards bandage. My mildest case went the whole time without treatment.

During the development of the disease the caput, collum and the acetabulum become more or less flattened and the name of coxa plana is just as characteristic at all the developmental stages of the disease as at the definite stage. In the earliest stages it is the flat epiphysis that first strikes the eye, and in the final stage it is the ordinary, extremely flattened caput and collum which characterizes the disease.

The development takes place slowly during several years and I have tried, in order to obtain more lucidity in the different phases of the development, to divide it into certain groups. 
I will here go briefly through these groups. Those who are interested in the matter can find them more fully described in Vol. 55 of Acta chirurgica Scandinavica.

\section{The developmental periods of the coxa plana}

I. The evolutionary period $3-4$ years.

a. The initial stage $1 / 2-1$ year. The epiphysis dense with decalcinated spots, flattened, uneven at the margin. The collum often with decaleinated spots just near the upper part of the epiphyseal line. The articular cartilage of normal height. Example, Fig. 4 a.

b. The fragmentation stage $2-3$ years. The epiphysis extremely flattened, divided. At the beginning it is often in three large pieces that can afterwards be divided into many small granules. Atrophy. Example, Fig 9 a, the beginning and $4 \mathrm{~b}$, the end of the fragmentation.

II. The healing period $1-2$ years. The epiphysis becomes homogeneous. The lime returns. (Example, Fig. 3. b.)

III. The growing period to the conclusion of the normal period of growth. During this the coxa plana assumes its final form.

IV. The definite stage.

In my paper in 1910 I described what I called the end-form, but added that I believed that a long observation period was necessary for, presumably, this end-form is subjected to further changes before it becomes definite. My few cases were then not under observation longer than to the close of the healing period or beginning of the period of growth. In this paper I can show that my then described end-form is really the definite form for most cases.

I consider that I am entitled to hold the opinion that those forms of coxa plana I here report are definite. I do so on the basis of having had them under observation for so long a period without the forms having changed. During the evolutionary stage the forms change, as we know, rather rapidly. During the healing period a change of form also takes place. In most cases the form is definite at the beginning of the period of growth, i. e. after the disease has existed 4-6 years. Thus Case 1 (Fig. 3), already in 1909 in the healing period (Fig. 3 b) and at the age of 9 years, was shown to have the same form as it now has (Fig. $3 \mathrm{c}$ ), i. e. during 13 years no change of form. In a delineation of the outlines both these contours appear delineated and it is seen how the lines of the caput in both cases run concentrically with each other. (see Fig. 1.)

Case 2 (Fig. 4) had attained in 1912, at an age of 12 years, the same 
form it now has, i. e. for 10 years it has had the same form. The deformation is here more advanced and it seems in these cases the definite form is reached later. In Case 3 (Fig. 5) the definite form had been attained in 1914 at an age of 15 years; as will be

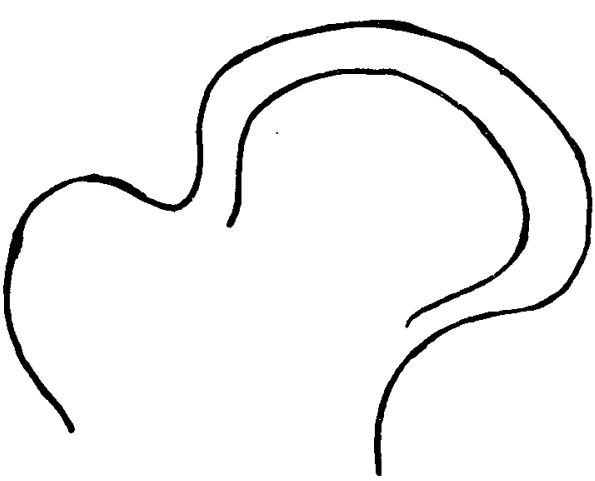

Fig. 1. seen, the deformation here is very severe and the picture then the same as Fig. 5 c.

I have chosen some typical roentgenograms of the different forms of the definite stage. They are to be found at the close and give, better than words, a picture of the varying appearances of the definite stage. Among them are to be found both extremes, minimal deformation (Fig. 9 b) and the most advanced deformation (Fig. 6 b). Both these extremes are different types of the final coxa plana. At the first glance one does not perhaps notice any characteristic common to them both, so different from each other do they appear; but, if one inspects them more closely, one will find an important common resemblance, the typical flattening. It looks as if the flattening had taken place through a pressure proceeding medially from above and coming into contact with a soft plastic caput and collum.

How this flattening has come about is difficult to say. The primary cause is an irregular cell proliferation in the cartilage and bone cells of the epiphysis and the collum with softening of caput and the medial part of the collum. The flattened form itself arises, I suppose, for the most part, from purely mechanical circumstances. In 1909 I sought to explain the genesis in this way, that the epiphysis and the medial portion of collum were softened, the articular cartilage was found like an envelope around the soft plastic substance and the pressure against the articular cavity flattened the caput and the immediate portion of the collum. The degree of flattening then depended on the degree and extent of the softening and the length of time that the caput and collum were, in this way, mechanically formable. It is seen how varying is the duration of time that elapses from the beginning of the flattening until its healing process starts.

In 1909 I described, moreover, how the caput can be pressed far out on the upper portion of the collum, almost up to the greater trochanter (for this case see Fig. 5 a). It seems to me as if this description should still hold good. 
If one now looks at the course of Shenton's line in these cases, one will find that it generally runs normally (see, for instance, Figs. $3 \& 7$ ), and this in spite of the caput-collum being shortened. The explanation of this lies in the fact that the contour of the lower collum is more curved and the more sharply curved according as the deformation is more pronounced. This curving of the collum is not the normal one. If, on the contrary, one endeavours to reconstruct the normal contour of the lower collum, starting from the little normally progressing part of the collum nearest the greater trochanter, one finds that the Shenton's line so made runs higher up. Shenton's line, during the whole development, apparently, can run normally, in spite of the fact that the caput early becomes lower in its entirety.

The flattening is thus the typical feature of a coxa plana even in the final stage. This flattening takes place in this wise, the caput is increased in breadth both upward and downward. A delineation of the outline with the deformed hip laid on the healthy one from Case 1 (Fig. 1) serves as a guide. The caput takes a pronounced egg form in the roentgen picture, viewed from the anterior aspect with the axis of the egg at an angle of $45^{\circ}$ against the horizontal and vertical plane. The broader pole of the egg lies downward-inward, its narrower pole upward-outward (éxample, see Figs. 3 c \& 4 c). The one long side is the flattened curve of the caput, the other long side one must conceive as passing through the collum. The broad axis inclines more than in a normal caput and is mostly dependent upon the upper portion of the collum being exceedingly shortened. The lower portion of the collum is also shortened, but not in such a high degree. At its seat of origin the collum is normal in its thickness, but the breadth is quickly increased forward towards the caput (éxample, Figs. $3 \mathrm{c} \& 3$ b). In some cases, the upper part of the caput lies quite close to the greater trochanter and then there is no collum to speak of above, only the seat of origin is to be seen (Fig. 4 c). The top of the caput will then lie with a large portion of the joint-surface outside of the articular cavity.

In order to get an actual picture of the appearance of the caput and the collum, I have also taken roentgenograms of my cases from a lateral view. The roentgenograms are taken in the flexion and abduction (method of LaUenstein), if it has been possible. On these pictures, then, the anterior and posterior outlines of the caput and collum are distinctly seen. If a frontal picture and a lateral picture are now placed side by side and viewed simultaneously, a stereoscopic picture of the caput and collum is obtained (see Figs. $3 \mathrm{c} \& \mathrm{~d}, 4 \mathrm{c} \& \mathrm{~d}, 5 \mathrm{c} \& \mathrm{~d}$ ). In the lateral picture the typical flattening is seen. Further, it is seen how, properly speaking, it is almost only the anterior portion of the caput 
that is enlarged; not only in the breadth but also its height is considerably greater. It extends, then, down towards the linea intertrochanterica and goes beyond it sometimes (Figs. $4 \mathrm{c} \& \mathrm{~d}$ ). In these more pronounced cases, the caput resembles, in a striking manner, a mushroom head with the collum as a mushroom foot. By these lateral pictures, moreover, an explanation is obtained as to how the top of the caput covers the trochanter major in the more pronounced cases. The caput lies, namely, in front of the trochanter and continues downward, extending forward surpassing the linea intertrochanterica. Therefore, in these advanced cases, it is not only the top of the collum which is practically wanting, but also the upper, anterior portion. The caput is therefore obliquely inserted in the collum. Its greatest extension is forward-upward, its smallest one backward-downward. Besides this, it inclines so that the centre of the periphery of the caput faces forwardupward.

On the frontal roentgen pictures a curved line is seen, in the severe cases, which passes - cutting the greater trochanter - down over the intertrochanter portion. If a frontal and a lateral picture are now viewed simultaneously, it will be found that this curved line tallies with the anterior periphery of the caput (Figs. $4 \mathrm{c} \& \mathrm{~d}$ )

I have previously pointed out how dissimilar the definite forms

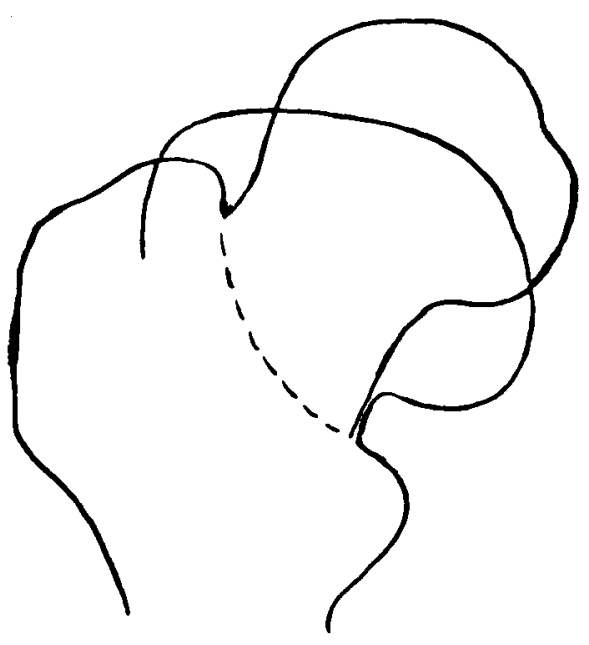

Fig. 2 . can be in the different cases in spite of the fundamental type of flattening being common to all. (compare, for example, Fig. $9 \mathrm{~b}$ with Fig. $6 \mathrm{~b}$ ). Between both these extremes we have Fig. $3 \mathrm{c}$ which most resembles Fig. $9 \mathrm{~b}$. My cases show, then, a serie of pictures, in different variations, between these five types which I have described. On closer inspection of my pictures there is, however, an essential difference between the types in Fig. 9 b \& Fig. $3 \mathrm{~b}$ and the type in Fig. $4 \mathrm{c}$ and the type in Figs. 6 \& 7 . They may be divided into three groups. These types may be said to be representatives of the three different groups. The two first mentioned are somewhat more normal, so to speak. A distinct caput and a distinct collum, separated from the trochanter, are to be distinguished. Of my 22 coxa plana cases with definite form, 14 belong to this type. It is, then, the more usual. I call it the ordinary type. 
In Case 2 (Fig. 4 c) there is nothing of the top of the caput to be found separated from the collum. The caput partially covers at the top and in front the greater trochanter. Nothing of the top part of the collum is to be seen. In the lateral picture (Fig. 4 d) the large anterior upper enlargement of the caput is seen. Fig. 2 shows the delineation of the normal and the deformed hips laid on each other from Fig. $4 \mathrm{c}$. Six of my cases belong to this second group.

The third embraces the most severe deformities. The caput does not lie in front of the greater trochanter. The collum is found shortened, but distinct even above. The upper pole of the caput is sharp at the margin and usually does not reach to the level of the greater trochanter (Fig. 6 b). Hereto belong 3 of the hip-joint cases (Figs. 3 c \& $6 \mathrm{~b}, 7)$ and, presumably, Fig. 8 from a 47 years old man.

The deformed joint-surface in the caput generally has an even, sharp contour in the roentgen picture but now and then it can, as in Fig. $6 \mathrm{~b}$, be uneven along the whole of its surface. The inequalities are so great that they must correspond to rather large hollows in the jointsurface. These must not, of course, be imagined to be only in the plane of the roentgen photograph but, here and there, on the whole of the jointsurface. The visible atrophic seats in the inside of the caput, which doubtless tally with hollows in other parts of the caput, favour this belief. Such are often found in arthritis deformans senilis.

In all these cases, where the deformity is distinctly pronounced, the hip-joint has more or less lost its character of ball-joint, with the possibility of movement in all directions. Often some direction of movement is wanting, e. g. abduction, and in the advanced cases, e. g. Figs. $5 \mathrm{~d}$, $6 \mathrm{~b} \& 7$, the caput is like a conical plug, articuiating with a similarly reformed articular cavity. The movement can then only take place in one plane, more immediately like the articulation of the mandible. The caput is, in its new form, too broad for the articular cavity. This reforms itself, certainly, as far as it can to suit the new form of the caput, but the greater or lesser portion of caput will lie outside of the articular cavity round about, but mostly above and in front. In the severe cases this part of the joint-surface never participates in the articulation; abduction is not possible. - Here above free bodies can be formed quite as in arthritis deformans. I have seen this in one case (Fig. 6 b). In one case I have seen an osteophyte from the acetabulum.

In spite of these considerable deformities in the hip-joint, the individual's capacity for motility is remarkably little reduced. One of my patients, Case 4, (Fig. $6 \&$ 7) with both hip-joints maximally deformed, and where the motility only consisted in flexion, could play football, cycle and dance. 
If this final form is that which afterwards lasts until life ends is more than I can say. Here again a long period of observation is needed, made in the way I have prescribed.

It appears to me likely that the forms I have described are definite in so far as they continue as long as the body is free from all regressive changes. Thus I should imagine that this form is permanent up to the age of 50. But my cases cannot give any proof of this before a quarter of a century.

It may be conceived from the fact that a part of the joint-surface of the caput never participates in the articulation that it may give rise to secondary deformations of the same nature as those found in arthritis deformans. I have had the apportunity of observing a few cases where the persons in question, men of about 50 years of age, came to me complaining of trouble from the hip: limping, aching, stiffness, and so on. (Fig. 8). They declared that the trouble had recently begun and did not know of any previous disease in the hip. On radiography I found in these cases a striking likeness to a severe case of coxa plana, although the deformation respecting the flattening of the caput had gone still further. The subjective symptoms in these cases have not been observed until, with the regressive changes in the hip-joint, the trouble forces them to go to the doctor. The examination yields the diagnosis arthritis deformans coxae, perhaps also senilis, but the considerable changes on the roentgen picture can impossibly have occurred in the short time the trouble has been felt, but rather it has existed long before. It is also possible that these cases have had a now-forgotten coxa plana in childhood that has not given any trouble before, when secondary changes of age begin, chiefly affecting, as is natural, a previously defective part of the body. In such cases where the changes in the joint are severe and resemble coxa plana, and the symptoms have not existed long, one can, with the greatest probability, make the diagnosis of coxa plana.

Roentgen pictures, resembling the final stage of coxa plana, may also result through a senile softening process just as they may be dependent on tuberculosis in childhood (Fig. 10 a, b) or a sustained septic infection, which soften the caput and collum.

The more pronounced the deformation becomes, the longer is the age period postponed when the definite stage can be reckoned on. The usual types, as in Figs. $3 \&$, attain their definite form during the close of the healing period and the beginning of the period of growth, the second group during the first part of the growing period, and the third group, the most severe, in the latter part of the growing period.

Cannot the definite type be prognosticated during the evolutionary stage, 
or at least cannot one say to which group it will belong? In some it is impossible. If decalcinated areas have appeared in the collum and if the epiphysis in the fragmentation stage is separated into small granules and extremely flattened, if, further, a part of the epiphysis lies outside of the articular cavity at the top of the collum and if, further, the upper part of the collum is extremely shortened, then, the definite form will belong to one of the two last groups. One can also be sure that those cases, where the epiphysis has been very little fragmented, dense with decalcinated spots, although it can be considerably flattened and uneven at the edge, will belong to the first group of the definite type.

\section{Summary}

The diagnosis coxa plana essentialis can with certainty be made only during the evolutionary stage. The definite form can therefore only be studied in cases that are followed from the beginning of the disease.

To endeavour to explain the definite form of coxa plana I have afterexamined those cases of coxa plana where I have been enabled to follow the development from the beginning until the end of the period of growth. There are shown to have been 22 hips; 10 of these have even attained an age of over 20 years.

The definite form, that I can hereby establish, is shown to be very variable in respect to the degree of the deformity, but the flattening of the caput, collum and acetabulum is common to all.

Through roentgenograms, both from a frontal and lateral view, one can obtain a plastic picture of the form of the caput and collum in the different cases. It can then be seen how it is mainly the anterior-superior portion of the caput that is enlarged and that this portion, in the more pronounced cases, lies outside of the articulation. With respect to the degree of the deformity, I have divided the definite coxa plana into three groups.

1. The caput preserves a rounded form. The caput and the collum can be well distinguished from each other and from the trochanter. (Ex., Figs. 3 d \& 9 b).

2. The upper and frontal part of the enlarged caput lies close to the greater trochanter. This portion of the caput is seen in the lateral picture, greatly enlarged, lying outside of the articulation. The upper part of the collum is not seen. (Ex., Fig. 4 d).

3. The joint-surface of the caput is uneven and more or less excavated. The upper pole of the caput is edgeformed and usually lower than the summit of the greater trochanter (Ex., Figs. 5 d, 6 b \& 7).

27 - Acta Radiologica. Iol. I. 1922. 


\section{Descriptions of the Figures}

Case 1. Gustaf C. Fig. 3. Tabula XXVI. On admittance to the hospital in 1907, 8 years old, he had limped for 8 months. Fig. 3 a, from 1907, shows beginning fragmentation and decalcinated spots in the collum. Fig. 3 b, from 1909, shows beginning healing. The definite torm had been attained. (Comp. Fig. 1). Fig. 3 c, from 1922, at 22 years of age, shows the definite stage from an anterior view and Fig. 3 d, from a lateral view. (In Lauenstein's position).

Case 2. Harald K. Fig. 4. Tabula XXVII. On admittance to the hospital in 1907, 7 years old, he had limped for 6 months. Fig. 4 a shows the initial stage in 1907. Decalcinated spots in the collum. Fig. 4 b, from 1908, shows the most severe degree of fragmentation. Fig. 4 c, from 1922, at 22 years of age, shows the definite stage from the frontal view and Fig. $4 \mathrm{~d}$, from the lateral view (Lauenstein's position).

Case 3. Erik A. Fig. 5. Tabula XXVIII. On admittance to the hospital in 1908, 13 years of age, he had had trouble for several years. - Fig. 5 a shows, in 1908 , the transition from the fragmentation stage to the healing stage. Fig. $5 \mathrm{~b}$, from 1911, shows how the form is on the way to become the definite one. Fig. $5 \mathrm{c}$, from 1922, at 28 years of age shows the definite stage from the frontal and Fig. 5, from the lateral view.

Case 4. Axel P. Figs. $6 \& 7$. Tabula XXIX. On admittance to the hospital in 1908, 8 years old, he had limped for 12 months. Fig. 6 a, from 1908, shows the fragmentation stage of the right hip with beginning pressing out of the caput outside of the articular cavity. Left hip normal. In 1910 he began to feel pain also in the left hip. Fig. 6 b shows, from 1922, the right hip at the age of 23 years. Observe the large concavities in the caput. Fig. 7 shows the left hip in 1922.

Case 5. Klas T. Fig. 8. Tabula XXIX. On admittance to the hospital he was 47 years old. Had limped for some years; pains had now started. Fig. 8 shows his roentgen picture - extreme deformation of the third type. I consider this to be a coxa plana essentialis.

Case 6. Folke A. Fig. 9. Tabula XXX. On admittance to the hospital in 1913, at 5 years of age, he had limped for 6 months. Fig. 9, from 1913, shows a beginning fragmentation; Fig. $9 \mathrm{~b}$ shows deformation that is very insignificant - only a slight flattening. The growth is certainly not at an end, but slight deformations become definite early.

Case 7. Harry S. Fig. 10. Tabula XXX. On admittance to the hospital in 1910 , at the age of 8 years, he had a typical coxitis. Fig. 10 shows his picture on admittance: strong atrophy of the joint, the cartilage thinned. This atrophy afterwards progressed and the disease developed as a typical coxitis: lay over one year in extension. When he was now, in 1922, after-examined he showed (Fig. 10 b) a picture that is a coxa plana; it cannot be distinguished from a coxa plana essentialis at this stage. It is therefore a coxa plana tuberculosa. If the pictures $10 \mathrm{~b}$ and $10 \mathrm{c}$ are more closely inspected, certain differences between this and a coxa plana essentialis can, however, be seen. Thus with this one the caput itself is enlarged in superior and anterior direction, bulging out towards the trochanter; even in this coxa plana tuberculosa a similar enlargement can be seen, but on closer inspection there is a distinct boundary between the caput and this enlargement which does not, then, belong to the caput. 


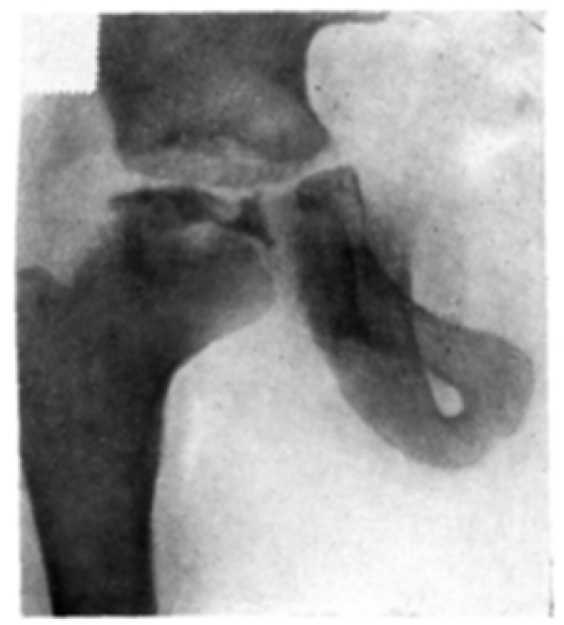

Fig. 3 a

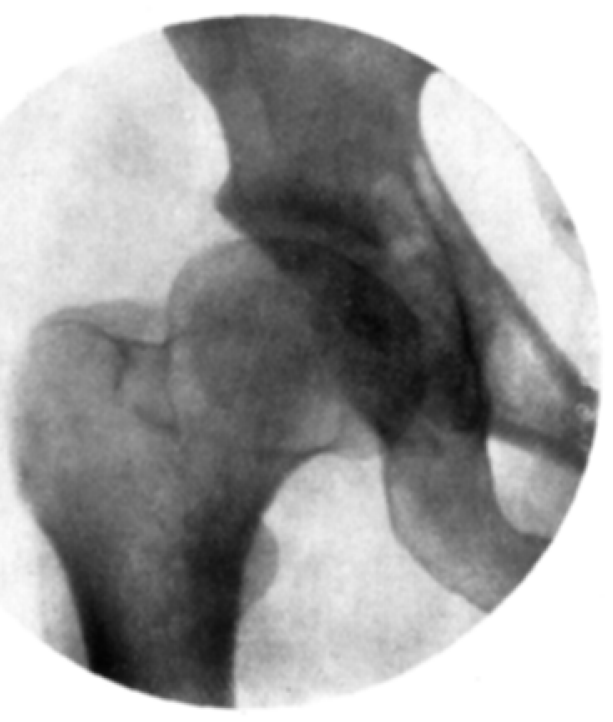

Fig. $3 c$

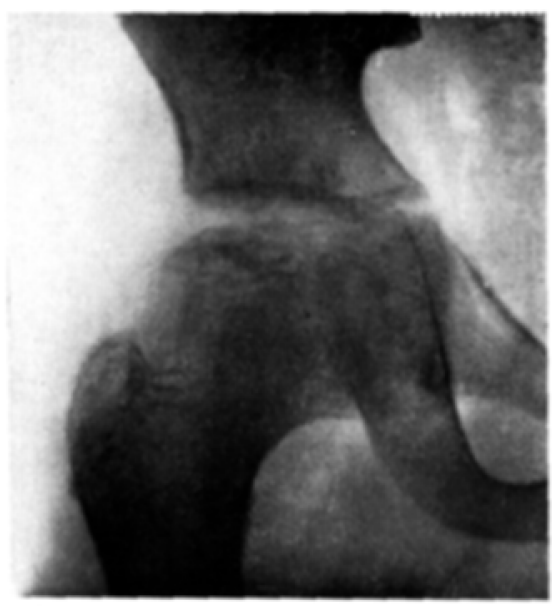

Fig. 3 b

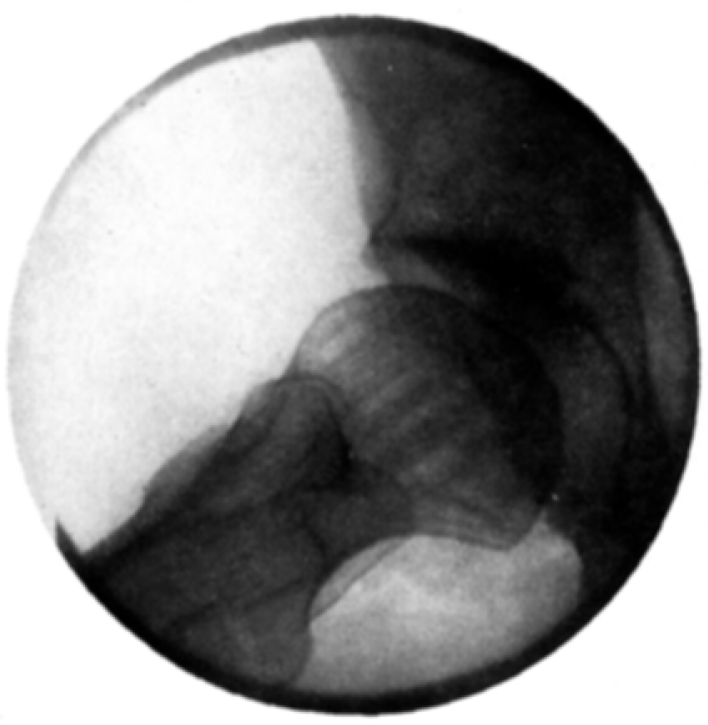

Fig. 3 d 


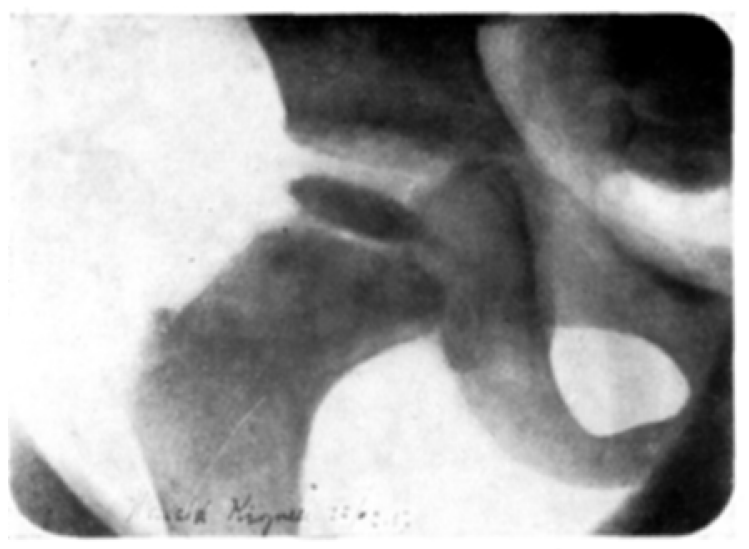

Fig. 4 a.

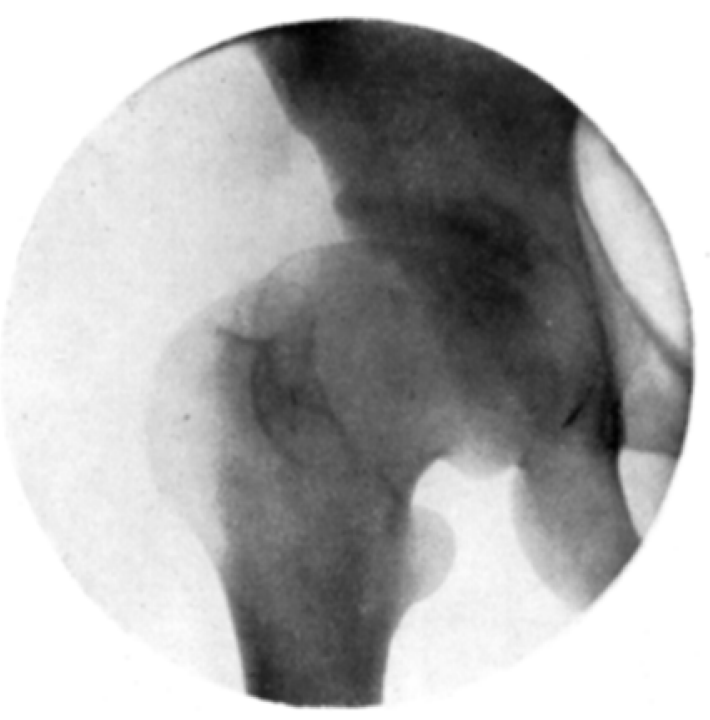

Fig. 4 c.

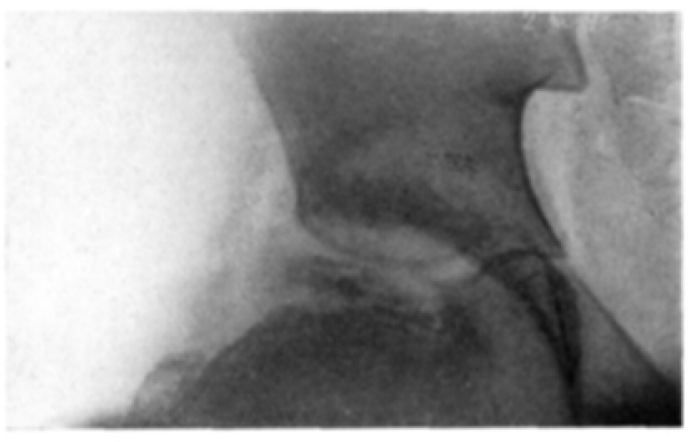

Fig 4 b.

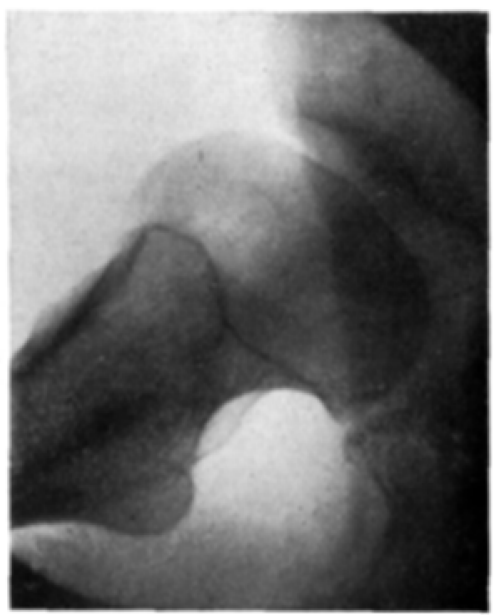

Fig. 4 d. 


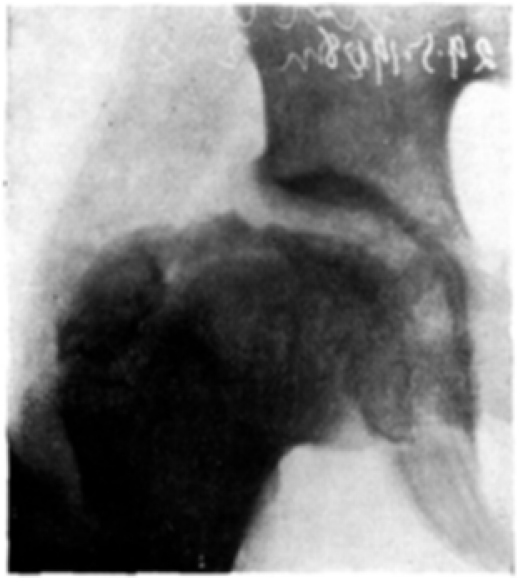

Fig. 5 a

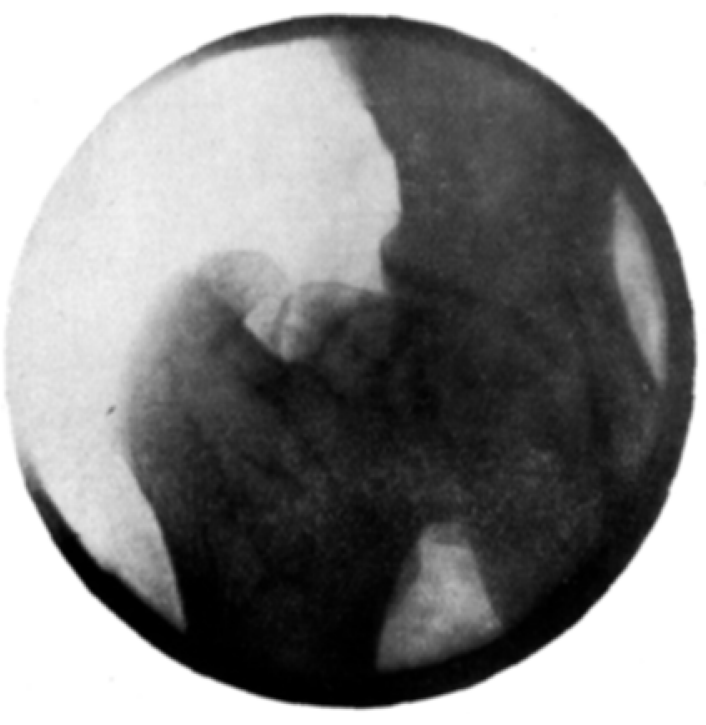

Fig. 5 c

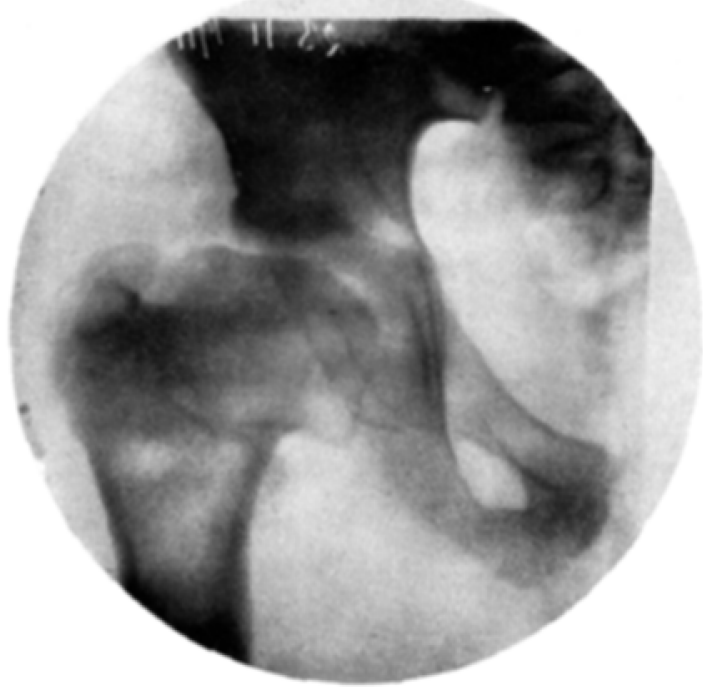

Fig. 5 b

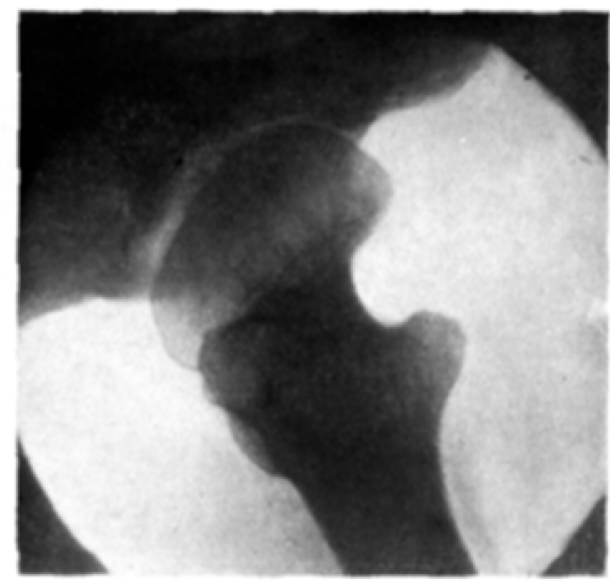

Fig. 5 d 


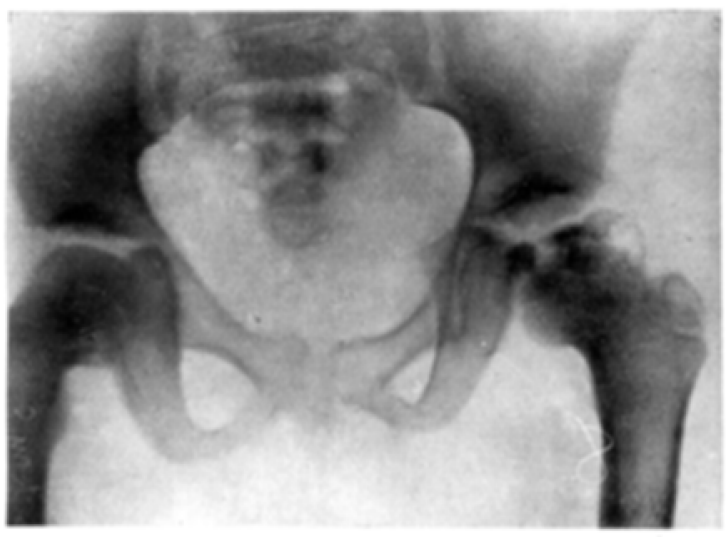

Fig. 6 a

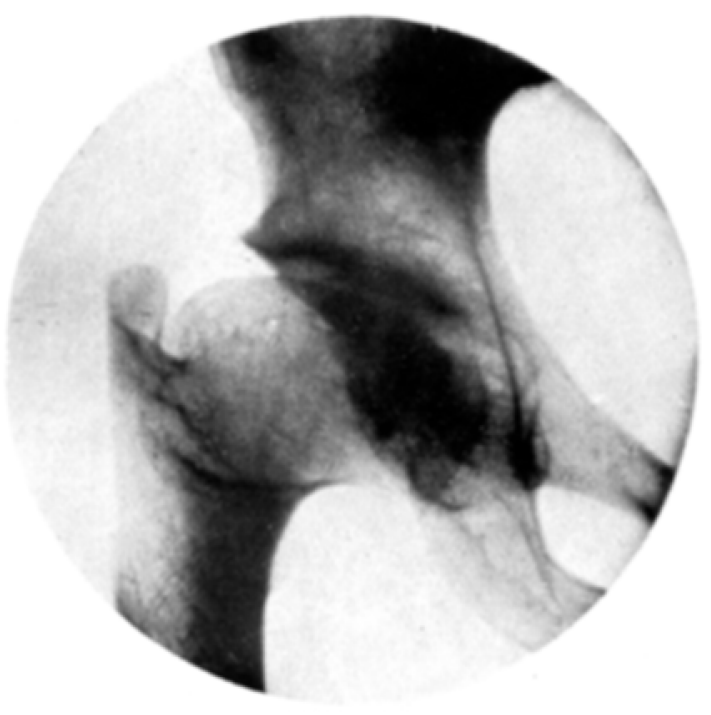

Fig. 7

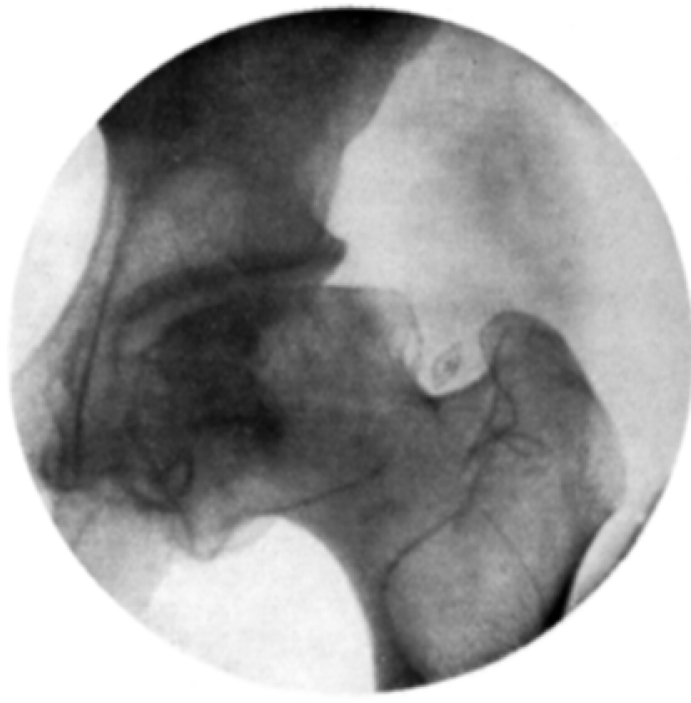

Fig. 6 b

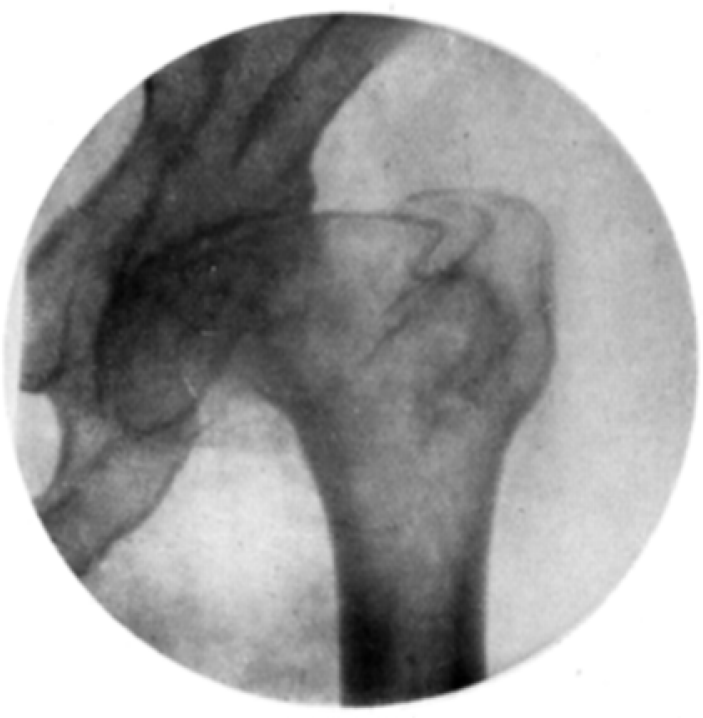

Fig. 8 


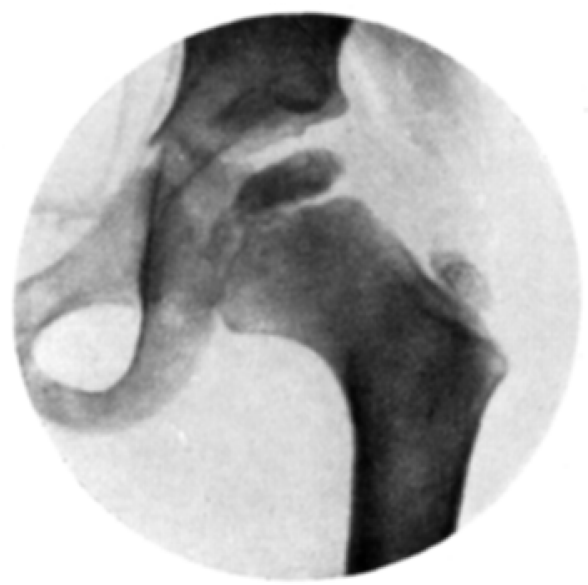

Fig. 9 a

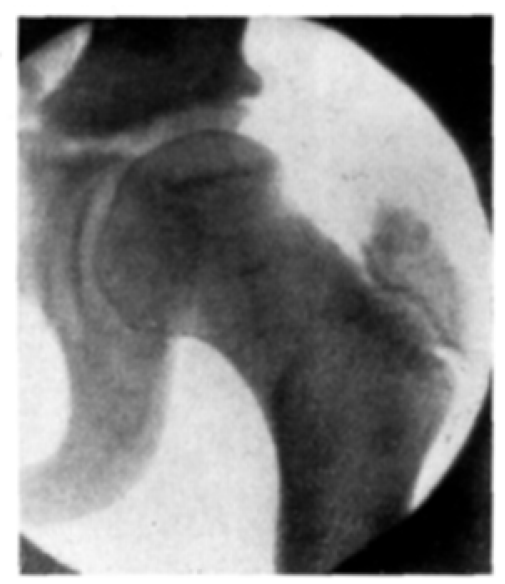

Fig. 9 b

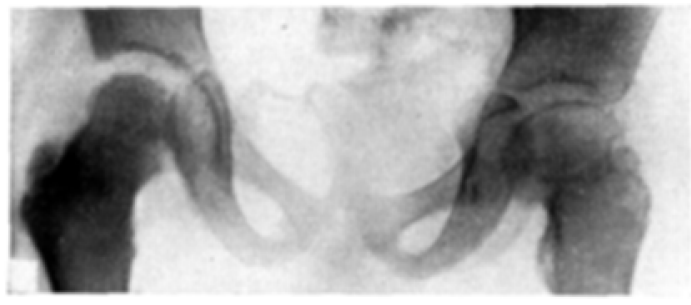

Fig 10 a

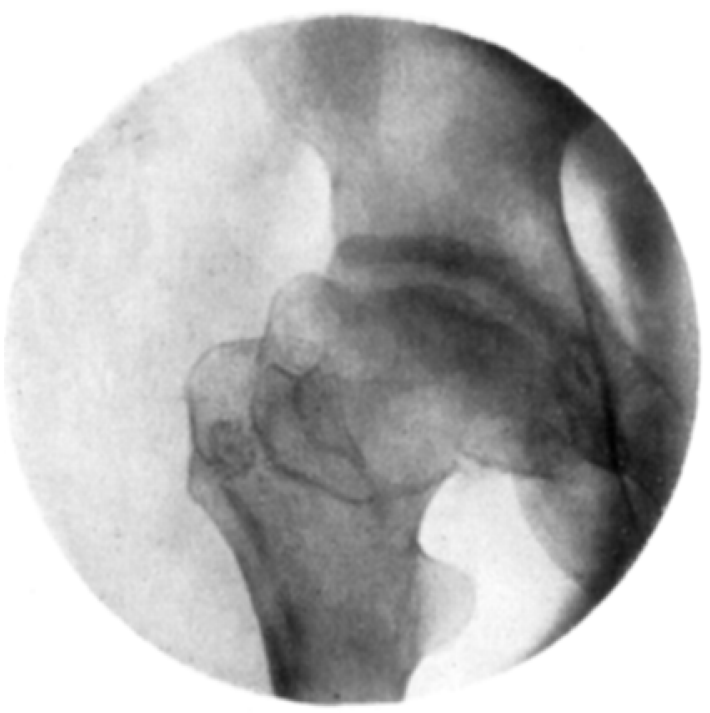

Fig. $10 \mathrm{~b}$

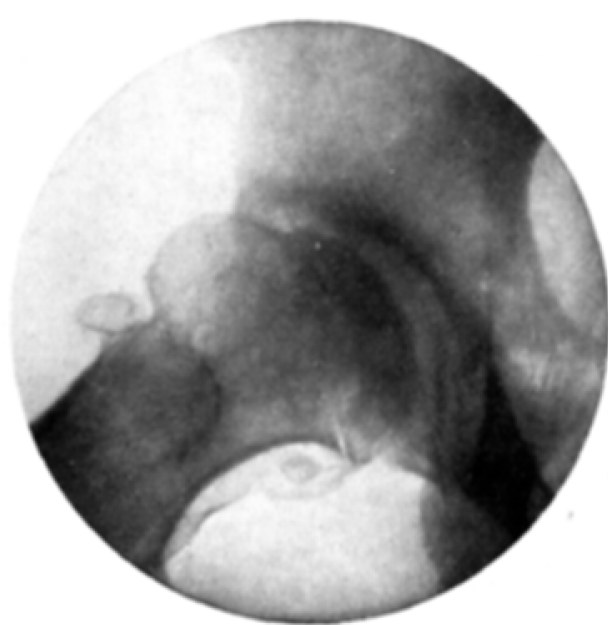

Fig. $10 \mathrm{c}$ 